\title{
Seroprevalence and immunological memory against SARS-CoV-2 in lung cancer patients: the SOLID study
}

\author{
Mariano Provencio $^{1 \wedge}$, Delvys Rodríguez-Abreu ${ }^{2}$, Ana L. Ortega ${ }^{3}$, Gloria Serrano ${ }^{4}$, Carlos Aguado $^{5}$, \\ Fernando Franco $^{1}$, Vanesa Gutierrez ${ }^{6}$, Guillermo López Vivanco ${ }^{7}$, María Guirado ${ }^{8}$, Gretel Benítez ${ }^{2}$, \\ Anna Estival $^{9}$, Virginia Calvo ${ }^{1 \wedge}$, Beatriz Jiménez ${ }^{10}$, Hugo Arasanz $^{11}$, Juan Coves ${ }^{12}$, Margarita Majem ${ }^{13}$, \\ Bartomeu Massutí1 ${ }^{14}$, Sergio Vázquez ${ }^{15}$, Oscar Juan-Vidal ${ }^{16}$, Ana Collazo-Lorduy ${ }^{1}$, Clara L. Gozálvez ${ }^{17}$, \\ Edel Del Barco $^{18}$, Adriana Rosero ${ }^{19}$, Joaquim Bosch-Barrerra ${ }^{20}$, María A. Moreno ${ }^{21}$, Xabier Mielgo-Rubio ${ }^{22}$, \\ José C. Villa ${ }^{23}$, Ana López-Martin ${ }^{24}$, Juan F. Córdoba ${ }^{25}$, Francisco de Asís Aparisi ${ }^{26}$, Marta Zafra ${ }^{27}$, \\ Joaquín Mosquera $^{28}$, Javier Pérez Altozano ${ }^{29}$, Ernest Nadal $^{30}$, Silvia Catot ${ }^{31}$, José Balsalobre ${ }^{32}$, \\ Teresa de Portugal $^{33}$, Paloma Martín ${ }^{34}$, Susana Cuesta de Juan ${ }^{35}$, Manuel Cobo ${ }^{6}$
}

${ }^{1}$ Medical Oncology Department, Hospital Universitario Puerta de Hierro Majadahonda, Madrid, Spain; ${ }^{2}$ Medical Oncology Department, Hospital Universitario Insular de Gran Canaria, Las Palmas, Spain; ${ }^{3}$ Medical Oncology Department, Hospital Universitario de Jaén, Jaén, Spain; ${ }^{4}$ Medical Oncology Department, Hospital Universiario Infanta Leonor, Madrid, Spain; ${ }^{5}$ Medical Oncology Department, Hospital Clínico San Carlos, Madrid, Spain; ${ }^{6}$ Medical Oncology Department, Hospital Universitario Regional de Málaga, Málaga, Spain; ${ }^{7}$ Medical Oncology Department, Hospital Universitario Cruces, Barakaldo, Spain; ${ }^{8}$ Medical Oncology Department, Hospital General Universitario de Elche, Alicante, Spain; ${ }^{9}$ Catalan Institute of Oncology, Hospital Universitari Germans Trias i Pujol, B-ARGO, IGTP, Badalona, Spain; ${ }^{10}$ Medical Oncology Department, Hospital Universitario HM Sanchinarro, Madrid, Spain; ${ }^{11}$ Medical Oncology Department, Complejo Hospitalario Navarra-Navarrabiomeed, Pamplona, Spain; ${ }^{12}$ Medical Oncology Department, Hospital Universitari Son LLàtzer, Palma de Mallorca, Spain; ${ }^{13}$ Medical Oncology Department, Hospital de la Santa Creu i Sant Pau, Barcelona, Spain; ${ }^{14}$ Medical Oncology Department, Hospital General Universitario de Alicante, Alicante, Spain; ${ }^{15}$ Medical Oncology Department, Hospital Universitario Lucus Augusti, Lugo, Spain; ${ }^{16}$ Medical Oncology Department, Hospital Universitario y Politécnico La Fe, Valencia, Spain; ${ }^{17}$ Medical Oncology Department, Hospital Universitari Sant Joan de Reus, Reus, Spain; ${ }^{18}$ Medical Oncology Department, Hospital Clínico Universitario de Salamanca, Salamanca, Spain; ${ }^{19}$ Medical Oncology Department, Hospital Universitario Infanta Cristina, Madrid, Spain; ${ }^{20}$ Medical Oncology Department, Institut Català d'Oncologia, Girona, Spain; ${ }^{21}$ Medical Oncology Department, Hospital Universitario de Puerto Real, Cádiz, Spain; ${ }^{22}$ Medical Oncology Department, Hospital Universitario Fundación Alcorcón, Madrid, Spain; ${ }^{23}$ Medical Oncology Department, Hospital General Universitario Ciudad Real, Ciudad Real, Spain; ${ }^{24}$ Medical Oncology Department, Hospital Universitario Severo Ochoa, Madrid, Spain; ${ }^{25}$ Medical Oncology Department, Hospital Universitario Arnau de Vilanova, Lleida, Spain; ${ }^{26}$ Medical Oncology Department, Hospital General Universitario de Valencia, Valencia, Spain; ${ }^{27}$ Medical Oncology Department, Hospital General Universitario Morales Messeguer, Murcia, Spain; ${ }^{28}$ Medical Oncology Department, Complejo Hospitalario Universitario A Coruña, A Coruña, Spain; ${ }^{29}$ Medical Oncology Department, Hospital Virgen de los Lirios, Alicante, Spain; ${ }^{30}$ Medical Oncology Department, Institut Català d’Oncologia, Hospitalet de Llobregat, Barcelona, Spain; ${ }^{31}$ Medical Oncology Department, Altaia, Xarxa Assistencial Universitaria Manresa, Barcelona, Spain; ${ }^{32}$ Medical Oncology Department, Hospital General Universitario Santa Lucia, Cartagena, Murcia, Spain; ${ }^{33}$ Medical Oncology Department, Complejo Hospitalario de Zamora, Zamora, Spain; ${ }^{34}$ Medical Oncology Department, Hospital Clínico Universitario de Valencia, Valencia, Spain; ${ }^{35}$ Laboratorio Central, Eurofins Megalab, Madrid, Spain

Contributions: (I) Conception and design: M Provencio; (II) Administrative support: None; (III) Provision of study materials or patients: None; (IV) Collection and assembly of data: D Rodríguez-Abreu, AL Ortega, G Serrano, C Aguado, F Franco, V Gutierrez, G López Vivanco, M Guirado, G Benítez, A Estival, V Calvo, B Jiménez, H Arasanz, J Coves, M Majem, B Massutí, S Vázquez, O Juan-Vidal, A Collazo-Lorduy, CL Gozálvez, E Del Barco, A Rosero, J Bosch-Barrerra, MA Moreno, X Mielgo, JC Villa, A López-Martin, JF Córdoba, F de Asís Aparisi, M Zafra, J Mosquera, J Pérez Altozano, E Nadal, S Catot, J Balsalobre, T de Portugal, P Martín, M Cobo; (V) Data analysis and interpretation: SC de Juan; (VI) Manuscript writing: All authors; (VII) Final approval of manuscript: All authors.

Correspondence to: Prof. Mariano Provencio; Dra. Virginia Calvo. Medical Oncology Department, Hospital Universitario Puerta de Hierro Majadahonda, Manuel de Falla 1, 28222 Majadahonda, Madrid, Spain. Email: mprovenciop@gmail.com; vircalvo@hotmail.com.

^ ORCID: Mariano Provencio, 0000-0001-9053-9197; Virginia Calvo, 0000-0002-3503-4847. 
Background: At present, we did not find any articles that studied seroprevalence and its persistence several months later in lung cancer patients in the setting of severe acute respiratory syndrome coronavirus 2 (SARSCoV-2) infection. Most patients with coronavirus disease 2019 (COVID-19) go on to develop antibodies (Abs) against viral proteins. However, it is not known how long these Abs last nor whether cancer treatments could affect the duration of immune response.

Methods: This prospective, longitudinal, multicenter serological study in the setting of SARS-CoV-2 infection was carried out in 50 Spanish hospitals. Eligibility criterion was the diagnosis of any lung cancer. The determination of anti-SARS-CoV-2 IgG Abs was performed by qualitative immuno-enzymatic assay using enzyme-linked immunosorbent assay (ELISA) kit from NovaLisa whose Abs target the recombinant antigen $\mathrm{N}$ of the nucleocapsid of SARS-CoV-2. The first Ab determination was performed between April 21 and June 3, 2020. The second Ab determination was performed in all previously seropositive patients, between September 10 and November 20, 2020. Study objectives were to prospectively determine seroprevalence in unselected lung cancer patients during the first wave of the pandemic; the persistence of immunity; protection or lack thereof against reinfection; and the influence of treatments on maintenance or loss of immunity.

Results: Of 1,500 patients, 128 were seropositive, overall prevalence of $8.5 \%$ seropositivity [95\% confidence interval (CI): 7.2-10.1\%]. Seventy-five percent were in active cancer treatment. Forty-seven point seven percent of IgG positive participants had experienced a symptomatic illness suspected of being infected with SARS-CoV-2 (95\% CI: 38.8-56.6\%). A second determination was performed on average 4.5 months later [interquartile range (IQR), 4.0-5.0 months] and obtained for 104 of the initially seropositive patients (81\%), it could not be obtained in 24 patients, the majority due to death caused by disease progression (73\%). In the second determination, IgG was not detected in $30.8 \%$ of patients. The severity of the infection, the need for hospitalization $(\mathrm{P}=0.032)$ and the presence of symptoms at diagnosis $(\mathrm{P}=0.02)$ were associated with persistence of immunity in the second determination. No variables or treatments received were associated with Abs loss.

Conclusions: Immunity against SARS-CoV-2 does not appear to be compromised by treatment and persists beyond 4 months. Neither do mortality rates appear to be particularly high in this unselected population.

Trial Registration: ClinicalTrials.gov identifier: NCT04407143.

Keywords: Immunity; lung cancer; severe acute respiratory syndrome coronavirus 2 (SARS-CoV-2); seroprevalence

Submitted Jun 20, 2021. Accepted for publication Oct 18, 2021.

doi: $10.21037 /$ tlcr-21-504

View this article at: https://dx.doi.org/10.21037/tlcr-21-504

\section{Introduction}

Coronavirus disease 2019 (COVID-19) is diagnosed by detecting the virus RNA with reverse transcriptionpolymerase chain reaction (RT-PCR). Most patients subsequently develop antibodies (Abs) against viral proteins. The degree to which this occurs varies and depends on when the sample is taken but is close to $100 \%$ (1). From the beginning of the COVID-19 pandemic, cancer patients, particularly those with lung cancer and hematological diseases, appeared to have higher morbidity and mortality than the general population (2), though not all series agreed (3). Selection bias may play a role in this since reported data are from patients visiting Accident and Emergency Departments or other medical services with COVID-19 symptoms, rather than from the entire population exposed to the virus (4). Even for the general population, we do not have clear information about patients' natural history. Here too, most studies are based on series of patients admitted to hospital, with mortality rates varying between $12.8 \%$ and $26 \%$ in Europe $(5,6)$.

With this in mind, the use of serological tests, without this selection bias, can help improve understanding of how 
COVID-19 behaves in the general population. In Spain, a seroprevalence sampling study has been carried out in the general population (7). However, no widespread serological screening policy has been introduced, still less in the population with cancer, and there are no associated clinical data for either population.

We reviewed the literature and found very few studies of seroprevalence in cancer patients with variable positivity (4/1,016 tested) (8). Neither did we find data on patients in whom infection was strongly suspected (9), or on highly selected populations with positivity over $50 \%$. The significant selection bias in these studies means they fail to represent the range of factors a study of these characteristics should reflect (10).

Severe acute respiratory syndrome coronavirus 2 (SARS$\mathrm{CoV}-2$ ) produces a detectable immune response in most cases. We think that lung cancer patients are qualified for assessing the seroprevalence and immunological memory against SARS-CoV-2 infection. A retrospective cohort study in patients with cancer who underwent SARS-CoV-2 testing has recently been published, but the seroconversion was different in specific patient groups (11). It is important testing SARS-CoV-2 Abs in patients with lung cancer because the duration of response and whether this protects against a second infection is unknown. In the largest study published to date on 121 plasma donors with different $\mathrm{Ab}$ determinations by ELISA, the last determination at 148 days showed a "slight drop" over time (12).

The Spanish Lung Cancer Group (SLGC) designed a prospective, multicenter study offering serological tests to lung cancer patients who attended oncology appointments, either for follow-up or treatment, with a second determination 4.5 months after the first, if this was positive. The study objectives were to prospectively determine seroprevalence in unselected lung cancer patients; their natural history; the persistence of immunity more than 4 months after the first determination; the protection or otherwise against reinfection after this period, and the nature of such protection; and the influence of treatments administered on maintenance or loss of immunity.

We present the following article in accordance with the STROBE reporting checklist (available at https://tlcr. amegroups.com/article/view/10.21037/tlcr-21-504/rc).

\section{Methods}

\section{Design}

This was a prospective, longitudinal, multicenter study offering serological tests to lung cancer patients who attended oncology appointments, either for follow-up or treatment, with a second determination 4.5 months after the first, if this IgG assessment was positive. It was designed by the SLCG. The study was approved by the Ethics Committee of Puerta de Hierro University Hospital on April 21, 2020. The trial was registered as NCT clinical trial Gov: NCT04407143 and conducted in accordance with the Declaration of Helsinki (as revised in 2013). All participants signed an informed consent form.

\section{Patients}

Patients were included in study from April 21, 2020, to June 3, 2020. One thousand five hundred determinations were distributed among Spain's autonomous communities (ACs) according to the population of each and the known incidence of COVID-19 at time of the beginning of the study. Fifty hospitals belonging to the SLCG network participated. The seroprevalence obtained in each AC was compared with that of the national seroprevalence study. A second determination was performed between September 10, 2020, and November 20, 2020, for those patients who had previously been seropositive. Data on symptoms, treatments received, evolution, follow-up of the patients and patients' clinical situation at the end of the study were collected from the medical records.

\section{Objectives}

To prospectively determine seroprevalence in unselected lung cancer patients during the first wave of the pandemic; the persistence of immunity; protection or lack thereof against reinfection; and the influence of treatments on maintenance or loss of immunity.

\section{Ab measurements}

Blood samples were collected in ethylenediaminetetraacetic acid (EDTA) tubes, centrifuged at 1,200 $\times \mathrm{g}$ for 15 minutes and transported to the Eurofins-Megalab central laboratory, Madrid, Spain. Determination of anti-SARS-CoV-2 IgG Abs was performed by qualitative immuno-enzymatic assay using ELISA kit from NovaLisa whose Abs target the recombinant antigen $\mathrm{N}$ of the nucleocapsid of SARS$\mathrm{CoV}-2$. Samples were processed with the following ELISA auto-analyzers: Thunderbolt (Gold Standard Diagnostics 
Table 1 Demographic characteristics

\begin{tabular}{|c|c|c|c|c|}
\hline Characteristics & All $(n=1,500)$ & Positive $(n=128)$ & Negative $(n=1,372)$ & $\mathrm{P}$ (overall) \\
\hline Age, median [IQR] & $66.0[60.0,72.0]$ & $67.0[61.0,73.0]$ & $66.0[60.0,72.0]$ & 0.139 \\
\hline Sex, n (\%) & & & & 0.791 \\
\hline Male & $1,020(68.0)$ & $90(70.3)$ & $930(67.7)$ & \\
\hline
\end{tabular}

SD, standard deviation; IQR, interquartile range.

Inc.), DSX (Palex Medical) and Analyzer (Euroinmun Diagnostics) using the following procedure and in accordance with manufacturers' instructions: samples were diluted 1:101 with the sample dilution buffer and $100 \mu \mathrm{L}$ of diluted sample/controls/calibrators were pipetted into the respective wells of the microtiter plate, which was then incubated for 1 hour at $37^{\circ} \mathrm{C}$. The plate was then washed 3 times with $300 \mu \mathrm{L}$ of washing solution to remove all unbound sample material and $100 \mu \mathrm{L}$ of horseradish peroxidase conjugate (HRP) was added to each well to bind to the Abs captured at the bottom of the well. The plate was then incubated for 30 minutes at room temperature before being washed again to remove unbound conjugate. Next, $100 \mu \mathrm{L}$ of tetramethylbenzidine substrate solution was added to each well and incubated for exactly 15 minutes at room temperature in the dark. A blue colored immune complex was formed. Finally, $100 \mu \mathrm{L}$ of stop solution (sulfuric acid) was added to each well, producing a change in color from blue to yellow. Extinction at 450/620 nm was measured photometrically. The intensity of this final product is proportional to the number of specific Abs in the sample.

The criteria for interpreting the results were as follows: ratio $<0.9$, negative; ratio $\geq 0.9$ and $\leq 1.1$, indeterminate; ratio $>1.1$, positive. The sensitivity of the NovaLisa kit is $89.7 \%$ [ $95 \%$ confidence interval (CI): 76.4-95.9\%] 2 weeks after positive RT-qPCR detection, and $91.2 \%$ (95\% CI: $77.0-97.0 \%) 3$ weeks after positive RT-qPCR detection (13-15). Specificity is $99.24 \%$ (95\% CI: $95.8-99.9 \%)$, determined in blood samples from donors collected before December 2019 in Germany and the USA. This procedure and analysis are in accordance with the requirements of the IVD Directive 98/79/EC of the European Parliament and Council of October 27, 1998, with regard to in vitro diagnostic medical devices (IVDs).

\section{Statistical analysis}

The possible relationship between the serology result (positive, negative or indeterminate) and the age or sex of the patients evaluated was studied using the Kruskal-Wallis and chi-square tests, respectively. Prevalences were estimated by calculating their $95 \% \mathrm{CI}$ using the Wilson method (overall prevalence) or the Clopper-Pearson method (prevalence in each AC). Prevalence in the different ACs was compared by independent chi-square test with continuity correction, with the $\mathrm{P}$ value estimated using the Monte-Carlo method (2,000 replications). The comparison of seroprevalence in this study with the values documented in the National Epidemiological Study of the Infection Caused by SARS-CoV-2 in Spain (ENE-COVID) (7) was performed by proportion comparison test in a sample with correction continuity (overall prevalence) or by binomial test (prevalences in each AC), assuming that the values of the ENE-COVID study are population parameters. Other comparisons of results (symptoms and contacts) were performed using the chisquare test, with continuity correction.

\section{Results}

\section{Characteristics of the evaluated patients}

One thousand five hundred patients were studied. Patients' age ranged between 26 and 89 years. Table 1 summarizes the demographic characteristics both in the total sample and by serology result. Age and sex distributions were similar in the two types of serological results, without statistically differences detected (Kruskal-Wallis test $\mathrm{P}=0.139$, and chisquare $\mathrm{P}=0.791$, respectively).

\section{Seropositivity prevalence $\left(\operatorname{Ig} G^{+}\right)$}

Of the 1,500 patients studied, 128 were seropositive, 
Table 2 General characteristics of patients with positive first IgG determination

\begin{tabular}{|c|c|}
\hline Positive IgG determination & $\mathrm{N}=128(\%)$ \\
\hline \multicolumn{2}{|l|}{ Symptoms at diagnosis } \\
\hline Asymptomatic & $69(53.9)$ \\
\hline Symptomatic & $59(46.1)$ \\
\hline \multicolumn{2}{|l|}{ Smoking habit } \\
\hline Former smoker ( $\geq 1$ year) & $80(62.5)$ \\
\hline Smoker & $29(22.7)$ \\
\hline Never smoker ( $\leq 100$ cigarettes/lifetime) & $15(11.7)$ \\
\hline Unknown & $4(3.1)$ \\
\hline \multicolumn{2}{|l|}{ PS (ECOG) } \\
\hline 0 & $35(27.3)$ \\
\hline 1 & $77(60.2)$ \\
\hline 2 & $13(10.2)$ \\
\hline 3 & $2(1.6)$ \\
\hline 4 & $1(0.8)$ \\
\hline \multicolumn{2}{|l|}{ Histology } \\
\hline SCLC & $11(8.6)$ \\
\hline NSCLC & 117 (91.4) \\
\hline Adenocarcinoma & $76(64.7)$ \\
\hline Squamous & $33(28.5)$ \\
\hline Others & $8(6.8)$ \\
\hline \multicolumn{2}{|l|}{ Number of cancer treatment lines } \\
\hline 1 & $2(1.6)$ \\
\hline 2 & $51(39.8)$ \\
\hline 3 & $24(18.8)$ \\
\hline 4 & $10(7.8)$ \\
\hline Other & $7(5.5)$ \\
\hline
\end{tabular}

PS, performance status; ECOG, Eastern Cooperative Oncology Group; SCLC, small-cell lung cancer; NSCLC, non-small-cell lung cancer.

representing an overall seropositivity prevalence of $8.5 \%$ (95\% CI: $7.2-10.1 \%$ ). Seropositivity prevalence was heterogeneous in the different ACs (chi-square, MonteCarlo $\mathrm{P}<0.001$ ) (Table S1). In the ENE-COVID study (7), the estimated prevalence of IgG Abs against SARS-CoV-2 was $5 \%$ in the first round and $5.2 \%$ in both the second and third rounds. In our study, this prevalence was $8.5 \%$, higher than the estimated values in any of the ENE-COVID rounds $(\mathrm{P}<0.001)$. In Castilla y León, Catalonia and the Community of Valencia, the seroprevalence observed in our study was either higher or much higher than in the ENECOVID study (Table S2).

\section{Characteristics of seropositive patients in the first determination}

Forty-seven point seven percent of IgG positive participants had experienced a symptomatic illness suspected of being SARS-CoV-2 infection (95\% CI: $38.8-56.6 \%$ ) of cases. Table 2 describes the main characteristics of patients positive for IgG in the first determination. For 11 patients, close contacts or relatives affected by COVID-19 were identified $(8.59 \%)$. In the case of affected relatives, the relationship was direct in all cases (woman: 7 patients; mother, daughter or sister: 1 patient in each case) except one (niece).

\section{Characteristics of seropositive patients in the second determination}

A second determination was performed on average 4.5 months later [interquartile range (IQR), 4.0-5.0 months] in 104 of the initially seropositive patients (81\%) (Table 3). A second sample was not obtained for 24 patients, mostly due to death from disease progression (73\%). Only $1(6.7 \%)$ was due to COVID-19. In the second determination, IgG was not detected in $30.8 \%(32 / 104)$ of the patients. Table 4 compares the two populations studied: those who maintained Abs in the second determination versus those who lost them. We found a statistically significant association between severity of the infection and the need for hospitalization $(\mathrm{P}=0.032)$, the presence of symptoms at diagnosis $(\mathrm{P}=0.02)$, fever $(\mathrm{P}=0.005)$ and nasal congestion $(\mathrm{P}=0.005)$ among them, and persistence of immunity in the second determination months later. No other variable was associated with $\mathrm{Ab}$ loss, even when comparing overall treatments administered or by grouping immunotherapy (IO) versus other treatments. Table 5 describes the treatments received, and Table $\mathrm{S} 3$ gives details of each drug administered. In $47 \%$ of patients ( $n=49)$, an increase in Abs was observed in the second determination compared to the first. The variation in IgG values was 0.5 [mean 0.5 (IQR, -0.2 to 1.2$)]$. We did not find any statistically significant differences in any of the parameters analyzed between patients who increased the Abs and those who maintained them (Table S4). No SARS-CoV-2 re-infections were 
Table 3 IgG results in the second determination

\begin{tabular}{lc}
\hline Results & $\mathrm{N}=104$ \\
\hline Time between tests (months), median [IQR] & $4.5[4,5]$ \\
IgG results & $2.8(1.2)$ \\
IgG values, mean (SD) & $2.6[1.9,3.7]$ \\
$\quad$ Median [IQR] & $0.5(1.0)$ \\
Variation between determination, mean (SD) & $0.5[-0.2,1.2]$ \\
$\quad$ Median [IQR] & $32(30.8)$ \\
Patients negative in the second test, $n(\%)$ & $72(69.2)$ \\
Patients positive in the second test, $\mathrm{n}(\%)$ & $23(22.1)$ \\
Patients who were positive without increase, $\mathrm{n}(\%)$ & $49(47.1)$ \\
Patients with increase in Abs, $\mathrm{n}(\%)$ &
\end{tabular}

IQR, interquartile range; SD, standard deviation; Abs, antibodies.

recorded. At time of last follow up, among those patients in whom a second determination was performed $89 \%$ (93/104) had completely recovered, without lasting effects, 9 were in the process of recovery $(8.7 \%), 1$ was in the process of recovery with lasting effects and 1 patient had been lost to follow-up.

\section{Discussion}

The seroprevalence rate in our study was $8.5 \%$, significantly lower than in other studies analyzing cancer patients infected with SARS-CoV-2, both in Spain (16) and abroad (17). This may be due to the larger sample size in our study. The prevalence generally agrees with that observed in the general population in Spain at the time, except in 2 ACs where the greater urban population in our sample could explain this. In more than $50 \%$ of cases, infection was not suspected and the patient was asymptomatic. Serological tests are particularly important to detect infection in asymptomatic patients as well as identify those who could potentially be protected against infection.

The mortality rate in our series is significantly different from that of other known series, probably because significant selection bias exists in those series. Only one of $128(0.8 \%)$ seropositive patients died from COVID-19 despite the fact that more than $30 \%$ required hospitalization for the virus, and that $75 \%$ were in active cancer treatment, the majority with several treatment lines.

There is little data on the duration of protection in the general population $(18,19)$. Some data on duration of immune response comes from analyses of other coronavirus infections, extrapolating these results to the current SARS$\mathrm{CoV}-2$ infection $(20,21)$. However, it is generally accepted that anti-SARS-CoV-2 Abs decay rapidly in persons with mild COVID-19 approximately 3 months after symptom onset (22).

In our study, $30.8 \%$ of all patients in whom a second determination was performed had serology negativization. To our knowledge, there are no comparable series in the published literature, particularly in lung cancer patients receiving aggressive treatments with combination chemotherapy (CT) or chemo-IO variations. Some data exist for the general population in 31 asymptomatic patients, $80 \%$ negativized Abs at 8 weeks (23). Also, data exist for 19 seropositive healthcare workers who had $58 \%$ seronegativization at 60 days (24). However, the sample sizes were significantly smaller than ours.

Our results allow us to be hopeful about the duration of protection against the virus, as well as make the same therapeutic efforts as for the general population since our patients' evolution does not seem to differ greatly.

Some selection bias may exist in our study since patients attended outpatient clinics rather than receiving palliative care. However, it is true that mortality was very low over 4 months later. Therefore, in the case of a serious infection, the same care should be available to these patients, including admission to intensive care.

In agreement with what we know about series in unselected populations, there is a relationship between severity of infection, the presence of fever or nasal 
Table 4 Baseline characteristics of seropositive patients in second determination

\begin{tabular}{|c|c|c|c|}
\hline Characteristics & Loss of IgG ( $n=32)$ & No loss of IgG $(n=72)$ & $P$ value \\
\hline Asymptomatic & $23(71.9)$ & $35(48.6)$ & \\
\hline Symptomatic & $9(28.2)$ & $37(51.4)$ & \\
\hline Age, median (SD) & $67.0(9.5)$ & $64.3(9.2)$ & 0.090 \\
\hline Male & $22(68.8)$ & $49(68.1)$ & \\
\hline Female & $10(31.3)$ & $23(31.9)$ & \\
\hline Smoking habits, n (\%) & & & 0.759 \\
\hline Active smoker & $5(15.6)$ & $9(12.5)$ & \\
\hline ECOG, n (\%) & & & 0.680 \\
\hline 0 & $8(25.0)$ & $23(31.9)$ & \\
\hline 1 & $22(68.8)$ & $43(59.7)$ & \\
\hline 2 & $6(8.3)$ & $2(6.3)$ & \\
\hline Histology, n (\%) & & & 0.222 \\
\hline Adenocarcinoma & $23(71.9)$ & $40(55.6)$ & \\
\hline Squamous & $6(18.8)$ & 17 (23.6) & \\
\hline Undifferentiated/NOS & $2(6.3)$ & $2(2.8)$ & \\
\hline Myalgia & $4(12.5)$ & $7(9.7)$ & 0.671 \\
\hline Anosmia & $3(9.4)$ & $5(6.9)$ & 0.668 \\
\hline Fatigue & $1(3.1)$ & $6(8.3)$ & 0.328 \\
\hline Dysgeusia & $2(6.3)$ & $4(5.6)$ & 0.889 \\
\hline Diarrhea & $0(0.0)$ & $4(5.6)$ & 0.174 \\
\hline Nasal congestion & $4(12.5)$ & $31(43)$ & $0.005^{\star}$ \\
\hline Headache & $0(0.0)$ & $3(4.1)$ & 0.241 \\
\hline Sore throat & $0(0.0)$ & $2(2.8)$ & 0.341 \\
\hline Congestion & $0(0.0)$ & $1(1.4)$ & 0.503 \\
\hline Driver mutation presented & $49(68.1)$ & $26(81.3)$ & 0.166 \\
\hline
\end{tabular}

Table 4 (continued) 
Table 4 (continued)

\begin{tabular}{|c|c|c|c|}
\hline Characteristics & Loss of lgG ( $n=32)$ & No loss of IgG $(n=72)$ & $P$ value \\
\hline CT & $30(43.5)$ & $28(47.6)$ & 0.207 \\
\hline 10 & $31(44.9)$ & $17(28.8)$ & 0.079 \\
\hline Hospitalization required for COVID-19 & $6(18.8)$ & $29(40.3)$ & $0.032^{*}$ \\
\hline Pneumonia & $6(18.8)$ & $14(19.4)$ & 0.934 \\
\hline Secondary infections & $0(0.0)$ & $2(2.8)$ & 0.341 \\
\hline Respiratory insufficiency & $6(18.8)$ & $10(13.9)$ & 0.526 \\
\hline Respiratory distress & $2(6.3)$ & $2(2.8)$ & 0.602 \\
\hline Coagulopathy & $0(0.0)$ & $1(1.4)$ & 0.503 \\
\hline ARDS & $2(6.3)$ & $1(1.4)$ & 0.172 \\
\hline Acute kidney injury & $1(3.1)$ & $2(2.8)$ & 0.922 \\
\hline Treatment delayed due to COVID-19 & $5(15.6)$ & $16(22.2)$ & 0.733 \\
\hline
\end{tabular}

*, P<0.05. SD, standard deviation; ECOG, Eastern Cooperative Oncology Group; CT, chemotherapy; IO, immunotherapy; COVID-19, coronavirus disease 2019; ARDS, acute respiratory distress syndrome.

congestion and persistence of Abs (11), without oncology treatments being a contributing factor in Ab loss over time. Neither did we detect any COVID-19 reinfections. It is not clear whether the low number of reinfections in seropositive patients is due to Ab levels, which even rose in $47 \%$ of patients in the second determination, or to their cellular immunity, which was not tested. Data from primates demonstrate that COVID-19 infection protects against reinfection for some time (25). It is likely that the $\mathrm{Ab}$ titer developed determines protection against reinfection just as in the rest of the population. This may also be the case with vaccination in cancer patients regardless of the treatments they receive. Even so, we believe it is imperative to develop long-term follow-up studies to understand the degree of protection and duration of titers obtained in serological determinations, particularly in populations such as cancer patients receiving immunosuppressive treatments and especially lung cancer following vaccination against COVID-19.

\section{Conclusions}

To our knowledge, our study is the first to be published that analyzes both seroprevalence in 1,500 lung cancer patients and the persistence of immunity several months after the first determination. Most of the patients included in our study were in active cancer treatment, over $30 \%$ with $\mathrm{IO}$, and the majority had received more than one line of treatment. There was only one death due to COVID-19 $(0.8 \%)$. We found no relationship between the treatments administered and loss of immunity against the virus. However, we found a relationship between the persistence of immunity and the severity of the infection, as observed in the general population. 
Table 5 Treatments received between the two serological determinations

\begin{tabular}{lc}
\hline Treatments & $\mathrm{N}(\%)$ \\
\hline Cancer treatment & $58(57.4)$ \\
Monotherapy & $43(42.6)$ \\
Combination & \\
Therapy type & $2(1.9)$ \\
Concurrent CT/RT & $2(1.9)$ \\
Adjuvant CT & $36(35.6)$ \\
CT (IV) & $10(9.9)$ \\
CT (IV) + IO & $2(1.9)$ \\
CT (IV) + oral targeted therapy & $1(0.9)$ \\
Oral CT & $22(21.8)$ \\
IO (anti-PD-1) & $14(13.9)$ \\
IO (anti-PD-L1) & $7(6.9)$ \\
Oral target therapy & $5(5.3)$ \\
Others* &
\end{tabular}

*, "others" includes cases treated with encorafenib + binimetinib, PD-1 agonist + soluble LAG-3 fusion protein, anti PD-L1 + monalizumab. CT, chemotherapy; RT, radiotherapy; IV, intravenous; IO, immunotherapy.

\section{Acknowledgments}

The authors thank all investigators who participated in the SOLID study: Dr. Pilar Diz (Complejo Asistencial Universitario de Leon), Dr. Rosa M. Villatoro (Hospital Costa del Sol), Dr. Pilar Lianes (Hospital de Mataro), Dr. M. Rosario Hernandez (Hospital Ntra. Sra Sonsoles), Dr. Juana Oramas (Hospital Univeristario de Canarias), Dr. Karmele Areses (Complejo Hospitalario e Ourense), Dr. Rafael Lopez (Hospital Clinico Universitario de Valladolid), Dr. Julio Ocaña (Hospital CIMA Sanitas), Dra. Maria Gonzalez Cao (Hospital Universario Quiron-Dexeus), Dr. Noemí Reguart (Hospital Clinic Barcelona), Dr. Manuel Fernandez (Hospital HM la Esperanza), Dr. Luis Enrique Chara (Hospital Universitario de Guadalajara), Dr. Judit Rubio (Hospital Univeristario de Mostoles), Dr. Alfonso Gurpide (Clinica Universitaria de Navarra), Dr. Ana Reyes Garcia (Hospital Universitario Rio Hortega, and Dr. Beatriz Esteban (Hospital General de Segovia). We also thank the patients, their families, all the participating clinical teams, and the Spanish Lung Cancer Group's staff for coordinating the study, and Roche Pharna for its support.

Funding: This study was supported by Roche Pharma and the European Union Horizon 2020 Research and Innovation Program under grant agreement No. 875160 CLARIFY project.

\section{Footnote}

Reporting Checklist: The authors have completed the STROBE reporting checklist. Available at https://tlcr. amegroups.com/article/view/10.21037/tlcr-21-504/rc

Data Sharing Statement: Available at https://tlcr.amegroups. com/article/view/10.21037/tlcr-21-504/dss

Conflicts of Interest: All authors have completed the ICMJE uniform disclosure form (available at https://tlcr.amegroups. com/article/view/10.21037/tlcr-21-504/coif). MP reports grants, personal fees and non-financial support from BMS, Roche and Astrazeneca and personal fees from MSD and TAKEDA and unrelated to the present study. CA reports grants, personal fees and non-financial support from Roche, personal fees and non-financial support from Pierre Fabre and personal fees from Astrazeneca, BMS, MSD and Novartis and unrelated to the present study. MG reports personal fees from Roche, MSD and BMS and unrelated to the present study. GB reports personal fees and nonfinancial support from Roche and AstraZeneca, personal fees from Sanofi and non-financial support from MSD, Novartis, BMS, MERCK and unrelated to the present study. AE reports personal fees from Roche and MSD and non-financial support from BMS, Lilly, Pharmamar and unrelated to the present study. VC reports personal fees from Roche, BMS, MSD, AstraZeneca and unrelated to the present study. HA reports personal fees from AstraZeneca and unrelated to the present study. JC reports personal fees from Roche, AstraZeneca, Boehringer, BMS, Novartis and Pfizer and unrelated to the present study. MM reports personal fees an non-financial supports from BMS, AstraZeneca, Boehringer and Roche, personal fees from Kyowa Kyrin, Pierre Fabre, Novartis and Sanofi and nonfinancial support from MSD and Lilly and unrelated to the present study. OJV reports personal fees from: Boehringer, BMS, MSD, Roche, AstraZeneca, Lilly and Takeda and unrelated to the present study. CLG reports personal fees from MSD; Novartis, Kyowa Karin and unrelated to the present study. JBB reports grant and personal fees from Pfizer, grant from Pierre Fabre, personal fees from Roche, 
MSD, BMS, AstraZeneca, Novartis and Boehringer and unrelated to the present study. XM reports grant and personal fees from BMS, personal fees and non-financial support from Roche, personal fees from AstraZeneca and unrelated to the present study. FdAA reports personal fees from Takeda, Mylan, Archimedes, Boehringer and unrelated to the present study. EN reports grant and personal fees from BMS and MERCK, personal fees from Roche, Pfizer, MSD, AstraZeneca, Lilly and Amgen and unrelated to the present study. SC reports personal fees and non-finacial support from Roche and personal fees from Boehringer and unrelated to the present study. The other authors have no conflicts of interest to declare.

Ethical Statement: The authors are accountable for all aspects of the work in ensuring that questions related to the accuracy or integrity of any part of the work are appropriately investigated and resolved. The study was approved by the Ethics Committee of Puerta de Hierro University Hospital on April 21, 2020. The trial was registered as NCT clinical trial Gov: NCT04407143 and conducted in accordance with the Declaration of Helsinki (as revised in 2013). All participants signed an informed consent form.

Open Access Statement: This is an Open Access article distributed in accordance with the Creative Commons Attribution-NonCommercial-NoDerivs 4.0 International License (CC BY-NC-ND 4.0), which permits the noncommercial replication and distribution of the article with the strict proviso that no changes or edits are made and the original work is properly cited (including links to both the formal publication through the relevant DOI and the license). See: https://creativecommons.org/licenses/by-nc-nd/4.0/.

\section{References}

1. Li Y, Yao L, Li J, et al. Stability issues of RT-PCR testing of SARS-CoV-2 for hospitalized patients clinically diagnosed with COVID-19. J Med Virol 2020;92:903-8.

2. Liang $W$, Guan $W$, Chen R, et al. Cancer patients in SARS-CoV-2 infection: a nationwide analysis in China. Lancet Oncol 2020;21:335-7.

3. Kuderer NM, Choueiri TK, Shah DP, et al. Clinical impact of COVID-19 on patients with cancer (CCC19): a cohort study. Lancet 2020;395:1907-18.

4. Garassino MC, Whisenant JG, Huang LC, et al. COVID-19 in patients with thoracic malignancies
(TERAVOLT): first results of an international, registrybased, cohort study. Lancet Oncol 2020;21:914-22.

5. Iaccarino G, Grassi G, Borghi C, et al. Age and Multimorbidity Predict Death Among COVID-19 Patients: Results of the SARS-RAS Study of the Italian Society of Hypertension. Hypertension 2020;76:366-72.

6. Nowak B, Szymański P, Pańkowski I, et al. Clinical characteristics and short-term outcomes of patients with coronavirus disease 2019: a retrospective single-center experience of a designated hospital in Poland. Pol Arch Intern Med 2020;130:407-11.

7. Pollán M, Pérez-Gómez B, Pastor-Barriuso R, et al. Prevalence of SARS-CoV-2 in Spain (ENE-COVID): a nationwide, population-based seroepidemiological study. Lancet 2020;396:535-44.

8. Berghoff AS, Gansterer M, Bathke AC, et al. SARS-CoV-2 Testing in Patients With Cancer Treated at a Tertiary Care Hospital During the COVID-19 Pandemic. J Clin Oncol 2020;38:3547-54.

9. Solodky ML, Galvez C, Russias B, et al. Lower detection rates of SARS-COV2 antibodies in cancer patients versus health care workers after symptomatic COVID-19. Ann Oncol 2020;31:1087-8.

10. Marra A, Generali D, Zagami P, et al. Seroconversion in patients with cancer and oncology health care workers infected by SARS-CoV-2. Ann Oncol 2021;32:113-9.

11. Thakkar A, Pradhan K, Jindal S, et al. Patterns of seroconversion for SARS-CoV2-IgG in patients with malignant disease and association with anticancer therapy. Nat Cancer 2021;2:392-9.

12. Wajnberg A, Amanat F, Firpo A, et al. Robust neutralizing antibodies to SARS-CoV-2 infection persist for months. Science 2020;370:1227-30.

13. Tré-Hardy M, Wilmet A, Beukinga I, et al. Analytical and clinical validation of an ELISA for specific SARSCoV-2 IgG, IgA, and IgM antibodies. J Med Virol 2021;93:803-11.

14. Schnurra C, Reiners N, Biemann R, et al. Comparison of the diagnostic sensitivity of SARS-CoV-2 nucleoprotein and glycoprotein-based antibody tests. J Clin Virol 2020;129:104544.

15. Yassine HM, Al-Jighefee H, Al-Sadeq DW, et al. Performance evaluation of five ELISA kits for detecting anti-SARS-COV-2 IgG antibodies. Int J Infect Dis 2021;102:181-7.

16. Cabezón-Gutiérrez L, Custodio-Cabello S, PalkaKotlowska M, et al. Seroprevalence of SARS-CoV-2specific antibodies in cancer outpatients in Madrid (Spain): 
A single center, prospective, cohort study and a review of available data. Cancer Treat Rev 2020;90:102102.

17. Liu T, Zeng G, Tao H, et al. Low prevalence of IgG antibodies to SARS-CoV-2 in cancer patients with COVID-19. Int J Cancer 2020;147:3267-9.

18. Guo L, Ren L, Yang S, et al. Profiling Early Humoral Response to Diagnose Novel Coronavirus Disease (COVID-19). Clin Infect Dis 2020;71:778-85.

19. To KK, Tsang OT, Leung WS, et al. Temporal profiles of viral load in posterior oropharyngeal saliva samples and serum antibody responses during infection by SARSCoV-2: an observational cohort study. Lancet Infect Dis 2020;20:565-74

20. Callow KA, Parry HF, Sergeant M, et al. The time course of the immune response to experimental coronavirus infection of man. Epidemiol Infect 1990;105:435-46.

21. Reed SE. The behaviour of recent isolates of human

Cite this article as: Provencio $M$, Rodríguez-Abreu D, Ortega AL, Serrano G, Aguado C, Franco F, Gutierrez V, López Vivanco G, Guirado M, Benítez G, Estival A, Calvo V, Jiménez B, Arasanz H, Coves J, Majem M, Massutí B, Vázquez S, Juan-Vidal O, Collazo-Lorduy A, Gozálvez CL, Del Barco E, Rosero A, Bosch-Barrerra J, Moreno MA, Mielgo-Rubio X, Villa JC, López-Martin A, Córdoba JF, de Asís Aparisi F, Zafra M, Mosquera J, Pérez Altozano J, Nadal E, Catot S, Balsalobre J, de Portugal T, Martín P, Cuesta de Juan S, Cobo M. Seroprevalence and immunological memory against SARSCoV-2 in lung cancer patients: the SOLID study. Transl Lung Cancer Res 2022;11(1):53-63. doi: 10.21037/tlcr-21-504 respiratory coronavirus in vitro and in volunteers: evidence of heterogeneity among 229E-related strains. J Med Virol 1984;13:179-92.

22. Ibarrondo FJ, Fulcher JA, Goodman-Meza D, et al. Rapid Decay of Anti-SARS-CoV-2 Antibodies in Persons with Mild Covid-19. N Engl J Med 2020;383:1085-7.

23. Milani GP, Dioni L, Favero C, et al. Serological followup of SARS-CoV-2 asymptomatic subjects. Sci Rep 2020;10:20048.

24. Patel MM, Thornburg NJ, Stubblefield WB, et al. Change in Antibodies to SARS-CoV-2 Over 60 Days Among Health Care Personnel in Nashville, Tennessee. JAMA 2020;324:1781-2.

25. Chandrashekar A, Liu J, Martinot AJ, et al. SARS-CoV-2 infection protects against rechallenge in rhesus macaques. Science 2020;369:812-7. 


\section{Supplementary}

Table S1 Seroprevalence of SARS-CoV-2 by AC

\begin{tabular}{lcc}
\hline AC & IgG positive & Prevalence [95\% Cl] \\
\hline Andalusia & $8 / 224$ & $3.6 \%[1.7 \%, 7.2 \%]$ \\
Balearic Islands & $0 / 45$ & $0.0 \%[0.0 \%, 9.8 \%]$ \\
Canary Islands & $2 / 114$ & $1.8 \%[0.3 \%, 6.8 \%]$ \\
Castilla La Mancha & $4 / 27$ & $14.8 \%[4.9 \%, 34.6 \%]$ \\
Castilla y León & $17 / 75$ & $22.7 \%[14.1 \%, 34.1 \%]$ \\
Catalonia & $26 / 230$ & $11.3 \%[7.7 \%, 16.3 \%]$ \\
Valencia & $13 / 180$ & $7.2 \%[4.1 \%, 12.3 \%]$ \\
Galicia & $1 / 71$ & $1.4 \%[0.1 \%, 8.7 \%]$ \\
Community of Madrid & $50 / 402$ & $12.4 \%[9.5 \%, 16.2 \%]$ \\
Murcia & $0 / 35$ & $0.0 \%[0.0 \%, 12.3 \%]$ \\
Navarra & $3 / 46$ & $6.5 \%[1.7 \%, 18.9 \%]$ \\
Basque Country & $4 / 51$ & $7.8 \%[2.5 \%, 19.7 \%]$ \\
\hline
\end{tabular}

SARS-CoV-2, severe acute respiratory syndrome coronavirus 2; AC, autonomous community; Cl, confidence interval.

Table S2 Regional distribution of seropositivity in Spain

\begin{tabular}{lccc}
\hline AC & Solid study & ENE-COVID (\%) & P value \\
\hline Andalusia & $8 / 224(3.6 \%)$ & 2.60 & 0.394 \\
Balearic Islands & $0 / 45(0.0 \%)$ & 2.30 & 0.628 \\
Canary Islands & $2 / 114(1.8 \%)$ & 2.30 & 1.000 \\
Castilla La Mancha & $4 / 27(14.8 \%)$ & 10.40 & 0.520 \\
Castilla y León & $17 / 75(22.7 \%)$ & 6.90 & $<.80$ \\
Catalonia & $26 / 230(11.3 \%)$ & 2.40 & 0.001 \\
Valencian Community & $13 / 180(7.2 \%)$ & 2.10 & $<0.001$ \\
Galicia & $1 / 71(1.4 \%)$ & 11.30 & 1.000 \\
Madrid & $50 / 402(12.4 \%)$ & 1.40 & 0.478 \\
Murcia & $0 / 35(0.0 \%)$ & 5.70 & 1.000 \\
Navarra & $3 / 46(6.5 \%)$ & 4.00 & 0.746 \\
Basque Country & $4 / 51(7.8 \%)$ & 0.146 & \\
\hline
\end{tabular}

Comparison between the results of the SOLID study in patients with lung cancer and the ENE-COVID population study (first analysis). AC, autonomous community. 
Table S3 Frequency of use of registered drugs

\begin{tabular}{|c|c|c|}
\hline Drugs & Cycles (n=101), n (\%) & Patients (n=96), n (\%) \\
\hline \multicolumn{3}{|l|}{ Oral target therapy } \\
\hline Afatinib & $1(1.0)$ & $1(1.0)$ \\
\hline Erlotinib & $1(1.0)$ & $1(1.0)$ \\
\hline Osimertinib & $5(5.0)$ & $4(4.2)$ \\
\hline Entrectinib & $1(1.0)$ & $1(1.0)$ \\
\hline \multicolumn{3}{|l|}{ CT } \\
\hline Etoposide & $6(5.9)$ & $6(6.2)$ \\
\hline Gemcitabine & $4(4.0)$ & $4(4.2)$ \\
\hline Docetaxel & $6(5.9)$ & $6(6.2)$ \\
\hline Paclitaxel & $12(11.9)$ & $12(12.5)$ \\
\hline Pemetrexed & $14(13.9)$ & $13(13.5)$ \\
\hline Topotecan & $1(1.0)$ & $1(1.0)$ \\
\hline Vinorelbine & $8(7.9)$ & $7(7.3)$ \\
\hline \multicolumn{3}{|l|}{10} \\
\hline Atezolizumab & $6(5.9)$ & $6(6.2)$ \\
\hline Nivolumab & $14(13.9)$ & $14(14.6)$ \\
\hline \multicolumn{3}{|l|}{ Antiangiogenic } \\
\hline Bevacizumab & $1(1.0)$ & $1(1.0)$ \\
\hline Nintedanib & $2(2.0)$ & $2(2.1)$ \\
\hline Ramucirumab & $1(1.0)$ & $1(1.0)$ \\
\hline
\end{tabular}

The percentages have been calculated based on the total number of cycles and patients. CT, chemotherapy; IO, immunotherapy. 
Table S4 Comparison between the population with maintenance or increase in Ab levels

\begin{tabular}{|c|c|c|c|}
\hline Characteristics & IgG increase $(n=49), n(\%)$ & Maintain IgG levels ( $n=23), n(\%)$ & $P$ value \\
\hline I & $2(3.9)$ & $2(8.7)$ & \\
\hline II & $2(3.9)$ & $1(4.4)$ & \\
\hline III & $7(14.3)$ & $7(30.4)$ & \\
\hline Unknown & $4(8.2)$ & $2(8.7)$ & \\
\hline Driver mutation presented & $34(69.4)$ & $15(65.2)$ & 0.723 \\
\hline Cancer treatment received & $37(75.5)$ & $17(73.9)$ & 0.884 \\
\hline CT & $24(66.7)$ & $8(34.8)$ & 0.173 \\
\hline Complications due to COVID-19 & $10(20.4)$ & $7(30.4)$ & 0.360 \\
\hline Pneumonia & $9(18.4)$ & $5(21.7)$ & 0.736 \\
\hline Secondary infections & $1(2.0)$ & $1(4.4)$ & 0.579 \\
\hline Respiratory insufficiency & $8(16.3)$ & $2(8.7)$ & 0.383 \\
\hline Respiratory distress & $1(2.0)$ & $1(4.4)$ & 0.579 \\
\hline Myocarditis & $0(0.0)$ & $0(0.0)$ & - \\
\hline Heart failure & $1(2.0)$ & $0(0.0)$ & 0.490 \\
\hline Coagulopathy & $0(0.0)$ & $1(4.4)$ & 0.142 \\
\hline
\end{tabular}

Ab, antibody; COVID-19, coronavirus disease 2019; CT, chemotherapy; IO, immunotherapy; ARDS, acute respiratory distress syndrome. 